QUIPURAMAYOC Revista de la Facultad de Ciencias Contables

Vol. 20 N.o 37 pp. 123-130 (2012) UNMSM, Lima, Perú

ISSN: 1560-9103 (versión impresa) / ISSN: 1609-8196 (versión electrónica)

\title{
MODELOS DE CALIDAD EN EL MARCO NORMATIVO DE LA ACREDITACIÓN DE LAS UNIVERSIDADES DEL PERÚ
}

\author{
QUALITY MODELS IN THE REGULATORY FRAMEWORK FOR THE \\ ACCREDITATION OF THE UNIVERSITIES OF PERU \\ Adrian Alejandro Flores Konja* \\ Docente Principal de la Facultad de Ciencias Contables - UNMSM
}

Colaborador: Manuel Alberto Hidalgo Tupia**

Docente Auxiliar de la Facultad de Ciencias Contables - UNMSM

[Recepción: Julio de 2012/ Conformidad: Agosto de 2012]

\section{RESUMEN}

EL presente artículo es resultado de una breve investigación exploratoria de la relación entre los Modelos de calidad propuestos por la doctrina administrativa y el proceso de acreditación nacional de las universidades peruanas. La universidad peruana, reflejando los cambios políticos, sociales, culturales y tecnológicos, está experimentando a su vez un proceso de cambio, por ejemplo, una gran expansión tanto en las carreras, modalidades y número de estudiantes. Sin embargo, este proceso de expansión, en muchos casos no planificada, ha creado serias dudas y cuestionamientos sobre si en la expansión se está garantizando la calidad de la formación profesional, la investigación y la extensión social. Además, debe ser coherente con las demandas sociales, del mercado y del desarrollo nacional. Para responder a esta necesidad, se han promovido un sistema de acreditación de la calidad de la enseñanza universitaria, a través de la creación de un Sistema Nacional de Evaluación, Acreditación y Certificación de la Calidad Educativa (Sineace), fue dispuesta en el año 2003 por la Ley General de Educación, 28044. Se desea conocer si los modelos de calidad propuestos en las normas de acreditación de dicho Sistema son coherentes con las nuevas tendencias doctrinarias organizacionales.

\begin{abstract}
This article is the result of a brief exploratory investigation of the relationship between quality models proposed by the administrative doctrine and the national accreditation process of Peruvian universities.

The Peruvian university, reflecting on the political, social, cultural and technological changes in our society, is also experiencing a process of change, for example, a major expansion in university departments, modalities and number of students. However, this expansion process, often unplanned, has created serious doubts and questions about whether the expansion is ensuring the quality of professional training, research and social outreach. It must also be consistent with the social demands of the market and national development. To meet this need, we have promoted a system of accreditation of the quality of university education through the creation of a National Assessment, Accreditation and Certification of Educational Quality (SINEACE), was prepared in 2003 by the General Law of Education, 28044. They want to know if the quality models proposed in the accreditation standards of the system are consistent with organization doctrinal trends.
\end{abstract}

* Doctor en Ciencias Contables y Empresariales, y Magister en Contabilidad con mención en Auditoría. Contador Público Colegiado Certificado. Actual Decano de la Facultad de Ciencias Contables -UNMSM. E-mail: afloreskonja@yahoo.es

** Magister en Ciencias Económicas. E-mail: hidalgoman@gmail.com 
Palabras claves: Calidad. Modelos de Calidad. Universidad Peruana. Formación Profesional. Acreditación de carreras universitarias. SINEACE. CONEAU. Administración Universitaria

\section{INTRODUCCIÓN}

La administración universitaria es un campo riquísimo, debido a la singularidad del "producto" de la organización: la formación profesional. Interrelacionada a la investigación, la extensión universitaria, y la proyección social. En este caso, los activos tangibles de la organización - la universidad - no tienen la importancia estratégica de sus valores intangibles, y en esto coincide con las tendencias señaladas por los nuevos enfoques administrativos, como lo son el cuadro de mando integral, especialmente, pero también el planeamiento estratégico, los sistemas de gestión de la calidad, la mejora continua, la formulación de indicadores y el capital intelectual. Si los enfoques administrativos centrados en la empresa manufacturera o comercial presentaban serias diferencias con la administración universitaria propiamente dicha, los nuevos enfoques están mucho más cercanos a la gestión universitaria que los anteriores.

Los nuevos enfoques administrativos han irrumpido en la gestión universitaria por dos fuentes: la propia conciencia profesional desde la cátedra, que gestiona las demandas sociales, de mercado y las más trascendentes, marcadas por la globalización, y por su parte la acción de la administración estatal, preocupada además por la calidad educativa ante la inusual explosión de la oferta educativa universitaria. El propio Ministerio de Educación (MINEDU, 2006: 56) relaciona "el aumento de las instituciones universitarias e deterioro de la calidad académica". Esta explosión responde a los procesos eco-
Keywords: Quality. Quality Models. Peruvian University. Training. Accreditation. SINEACE. CONEAU. University Administration.

nómicos (crecimiento económico), sociales (aumento de expectativas de desarrollo personal), demográficos (urbanización de la población peruana) y tecnológicos, que han impactado en una demanda no atendida de formación universitaria, sin que se pueda tener una certeza sobre la calidad de la nueva oferta educativa en su conjunto.

Estas tendencias se han reflejado en los procesos de autoevaluación y de acreditación de las carreras universitarias, que han sido realizadas en forma espontánea por las universidades más prestigiosas, inclusive como acreditación internacional, pero que en los próximos años se impone como un proceso mandatorio dentro del Sistema Nacional de Aseguramiento de la Calidad Educativa, SINEACE. Estos procesos se están realizando en base a los nuevos enfoques organizacionales mencionados, como los Modelos de Calidad, planificación estratégica, formulación de indicadores y Cuadro de Mando Integral. Por ejemplo, la Universidad Nacional del Callao declara formalmente que su Plan Estratégico vigente se formula al Cuadro de Mando Integral.

Enfocamos nuestra atención a un tipo de organización específica, la universidad pública, cuya particularidad reside en el hecho el propietario de la misma es el Estado, siendo que, además de la doctrina administrativa, y de las normas internacionales, existen normas legales comunes dictadas por el propio Estado, normas que determinan su actuación y que cambian de acuerdo al entorno político y social del país.

En materia de las universidades públicas, la principal norma externa es la Ley Univer- 
sitaria, fijando los objetivos y fines, el marco organizativo básico y las relaciones entre los diferentes actores de la institución y las organizaciones externas.

Adicionalmente, existen normas dictadas por la Asamblea Nacional de Rectores, específicamente el Sistema Nacional de Evaluación, Acreditación y Certificación de la Calidad Educativa, SINEACE, en especial del órgano correspondiente, el Consejo de Evaluación, Acreditación y Certificación de la Calidad de la Educación Superior Universitaria.(CONEAU)

La mayor parte de estas normas se dirigen al aseguramiento de la calidad educativa y de la calidad del servicio administrativo, e incluyen los modernos instrumentos de la planificación estratégica que son requeridos, tanto nacional como internacionalmente. En este sentido, las Universidades poseen instrumentos de gestión tales como: Plan de Desarrollo Institucional, Plan de Desarrollo Estratégico, Planes Operativos Institucionales Anuales, Presupuesto Institucional, entre muchos otros.

\section{GESTION UNIVERSITARIA SEGÚN LA LEY UNIVERSITARIA}

El logro de los fines de la Universidad, como se definen en la propia Ley Universitaria Ley No 2373, del 9 de diciembre de 1983, y sus sucesivas modificaciones, es lo que constituye la gestión universitaria. Allí también se norma las relaciones con los diferentes actores, como los docentes, estudiantes y trabajadores, entre otros que se llamarían ahora "grupos de interés", constituyéndose en un instrumento vital para analizar la gestión en sí, en este caso de las Universidades Publicas de Perú.

El Artículo 2, señala los fines de las Universidades: a) Conservar, acrecentar $y$ transmitir la cultura universal con sentido crítico y creativo afirmando preferentemente los valores nacionales;

b) Realizar investigación en las humanidades, las ciencias y las tecnologías, y fomentar la creación intelectual y artística;

c) Formar humanistas, científicos y profesionales de alta calidad académica, de acuerdo con las necesidades del país, desarrollar en sus miembros los valores éticos y cívicos, las actitudes de responsabilidad y solidaridad social y el conocimiento de la realidad nacional, así como la necesidad de la integración nacional, latinoamericana y universal.

d) Extender su acción y sus servicios a la comunidad y promover su desarrollo integral".

Se trata así de la formación profesional, de la investigación científica y de la proyección social.

\section{Sistemas de Gestión de la Calidad como Modelos de Calidad}

Como en la generalidad de los casos en gestión, el concepto de calidad es central, también en el presenta caso, concibiéndose la calidad como la coincidencia del debe ser con la realidad con respecto a un bien o servicio que brinda la organización y su capacidad de satisfacer al cliente. En nuestro caso es el servicio de enseñanza aprendizaje, que también se extiende a la investigación, a la proyección social y la extensión universitaria. Algunas orientaciones como las que citamos conciben la formación profesional como la base la enseñanza aprendizaje, la investigación, la extensión universitaria y la proyección social como componentes de la formación profesional.

En tal sentido, las tendencias administrativas modernas conciben Sistemas de Ca- 
lidad, o Sistemas de Gestión de Calidad, las que se han instaurados hacen varias décadas en los sectores manufactureros y de servicios públicos tangibles y en los años recientes en la educación. Se han propuesto normas de calidad y normas de aseguramiento de la calidad, como las ya muy conocidas normas ISO, y, como la Calidad esta relacionada directamente a la satisfacción del cliente, siendo el cliente uno de los grupos de interés más importantes a considerarse con respecto a la Responsabilidad Social.

Además, es muy importante hacer notar que el proceso de Acreditación de las Carreras Universitarias se hace, como los establece los organismos competentes, en base a Modelos de Calidad establecidos de acuerdo a lo más avanzado de las doctrinas administrativas. Al respecto, el CONEAU, Consejo de Evaluación, Acreditación y Certificación de la Calidad de la Educación Superior Universitaria, organismo rector de la acreditación universitaria, ha publicado su modelo de Calidad para la Acreditación de las Carreras Universitarias y Estándares para las Carreras de Educación.

\section{Como se define la Calidad en la Educa- ción Superior Universitaria}

Según el Consejo de Evaluación, Acreditación y Certificación de la Calidad de la Educación Superior Universitaria, (CONEAU, 2008, 10-11)), la calidad, dentro del marco legal vigente, es: "el conjunto de características inherentes a un producto o servicio que cumple los requisitos para satisfacer las necesidades preestablecidas. Así una carrera universitaria de calidad define claramente su misión o propósito en función de sus grupos de interés, estos propósitos abarcan las actividades confiadas por la sociedad."

CONEAU (loc. Cit) cita la Declaración mundial sobre la Educación Superior en el siglo XXI, que "amplía esta definición en cuanto a su evaluación, involucrando más categorías de análisis:

"La calidad de la enseñanza superior es un concepto pluridimensional que debería comprender todas sus funciones y actividades: enseñanza y programas académicos, investigación y becas, personal, estudiantes, edificios, instalaciones, equipamiento y servicios a la comunidad y al mundo universitario. Una autoevaluación interna y un examen externo realizados con transparencia por expertos independientes, en lo posible especializados en lo internacional, son esenciales para la mejora de la calidad. Deberían crearse instancias nacionales independientes, $y$ definirse normas comparativas de calidad, reconocidas en el plano internacional. Con miras a tener en cuenta la diversidad y evitar la uniformidad, debería prestarse la atención debida a las particularidades de los contextos institucional, nacional y regional. Los protagonistas deben ser parte integrante del proceso de evaluación institucional”.

\section{Continúa la cita (loc.cit):}

"La calidad requiere también que la enseñanza superior esté caracterizada por su dimensión internacional: el intercambio de conocimientos, la creación de sistemas interactivos, la movilidad de profesores y estudiantes y los proyectos de investigación internacionales, aun cuando se tengan debidamente en cuenta los valores culturales y las situaciones nacionales".

CONEAU (loc. Cit) también señala cuales son los elementos para lograr y mantener la calidad a nivel de pautas nacionales, regionales y globales, "principalmente la selección esmerada del personal y su perfeccionamiento constante, en particular mediante la 
promoción de planes de estudios adecuados para el perfeccionamiento del personal universitario, incluida la metodología del proceso pedagógico, y mediante la movilidad entre los países y los establecimientos de enseñanza superior y entre los establecimientos de educación superior y el mundo del trabajo, así como la movilidad de los estudiantes en cada país y entre los distintos países"

Añade que "las nuevas tecnologías de la información constituyen un instrumento im- portante en este proceso debido a su impacto en la adquisición de conocimientos teóricos y prácticos".

\section{Acreditación Universitaria y Modelos de Calidad de la Educación}

$\mathrm{Al}$ respecto, el CONEAU, Consejo de Evaluación, Acreditación y Certificación de la Calidad de la Educación Superior Universitaria, organismo rector de la acreditación universitaria, ha publicado su modelo de

\section{Gráfica $\mathbf{N}^{\circ} \mathbf{1}$}

\section{Modelo de calidad para la Acreditación de Carreras Universitarias}

Gestión de la Carrera

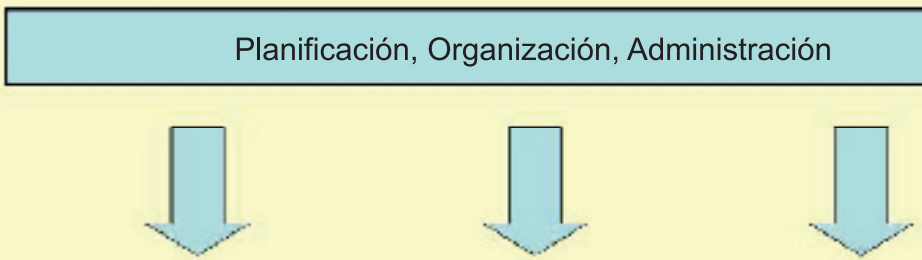

Formación Profesional

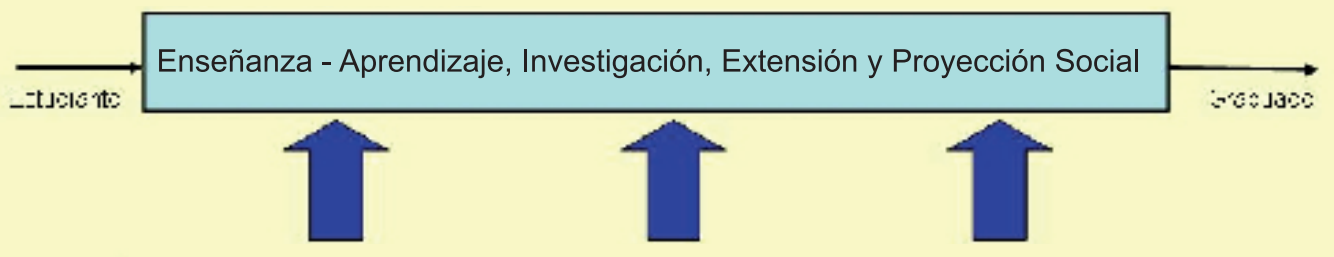

Servicios de Apoyo para la Formación Profesional

\begin{tabular}{|c|c|c|c|c|}
\hline Docentes & $\begin{array}{l}\text { Recursos } \\
\text { Financieros }\end{array}$ & Bienestar & 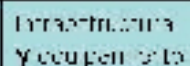 & $\begin{array}{l}\text { Grupos de } \\
\text { Interes }\end{array}$ \\
\hline
\end{tabular}

Fuente: CONEAU. Modelo de Calidad para la Acreditación de carreras Universitarias. 2008. 18

Calidad para la Acreditación de las Carreras

Universitarias y Estándares para las Carreras de Educación.

El modelo esta representado en la siguiente Gráfica

EL modelo de calidad para la Acreditación de Carreras Universitarias de CONEAU, como se puede apreciar en el gráfico adjunto, tiene una dimensión central, que es la de la Formación Profesional, y dos dimensiones auxiliares, como son la Gestión de la
Carrera y los Servicios de Apoyo para la formación profesional. A estas dimensiones se señalan los factores, y criterios por cada factor.

El aporte central de CONEAU en este Modelo es que establece los indicadores, adosando indicadores a los criterios, y adicionalmente, los indicadores con sus respectivas fuentes de verificación. En esto, el modelo está completamente alineado con el reciente énfasis en la doctrina administrativa 
en la necesidad de formular indicadores para evaluar los diversos aspectos de la gestión.

El antecedente al Modelo de CONEAU, es el de la Comisión Nacional de Autorización de Nuevas Universidades, el cual se basa en 11 Secciones, indicando criterios de evaluación en cada una de las 11 Secciones, pero planteando indicadores únicamente cuali- tativos, de atributo, y formulados genéricamente, salvo excepciones. Sin embargo, hay que decir que CONAFU no evalúa carreras profesionales, como CONEAU, sino universidades en su conjunto, lo que podría dificultad la formulación de indicadores generales.

En el siguiente Cuadro $n^{\circ} 1$ se registran dichos elementos, como se aprecia:

\section{Cuadro 1: DIMENSIONES, FACTORES, CRITERIOS PARA LA ACREDITACION DE CARRERAS UNIVERSITARIAS}

\begin{tabular}{|c|c|l|}
\hline DIMENSION & FACTOR & CRITERIO \\
\hline \multirow{2}{*}{$\begin{array}{c}\text { Gestión de la } \\
\text { Carrera }\end{array}$} & $\begin{array}{c}\text { Planificación, } \\
\text { organización, } \\
\text { dirección y control }\end{array}$ & Planificación estratégica. \\
\cline { 3 - 3 } & & organización, dirección y control \\
\hline
\end{tabular}

\begin{tabular}{|c|c|c|}
\hline DIMENSIÓN & FACTOR & CRITERIO \\
\hline \multirow{7}{*}{$\begin{array}{l}\text { Formación } \\
\text { Profesional }\end{array}$} & \multirow{5}{*}{$\begin{array}{l}\text { Enseñanza - } \\
\text { Aprendizaje }\end{array}$} & Proyecto Educativo. Currículo. \\
\hline & & Metodología de la Enseñanza - Aprendizaje. \\
\hline & & $\begin{array}{l}\text { Desarrollo de las actividades de Enseñanza - } \\
\text { Aprendizaje. }\end{array}$ \\
\hline & & Evaluación del Aprendizaje y acciones de mejora. \\
\hline & & Estudiantes y egresados. \\
\hline & Investigación & Generación y evaluación de proyectos de Investigación \\
\hline & \begin{tabular}{|l|} 
Extensión \\
Universitaria y \\
Proyección Social.
\end{tabular} & $\begin{array}{l}\text { Generación y evaluación de proyectos de Extensión } \\
\text { Universitaria y Proyección Social. }\end{array}$ \\
\hline
\end{tabular}




\begin{tabular}{|c|c|c|}
\hline DIMENSION & FACTOR & CRITERIO \\
\hline \multirow{8}{*}{$\begin{array}{l}\text { Servicios de } \\
\text { Apoyo para la } \\
\text { Formación } \\
\text { Profesional }\end{array}$} & \multirow{4}{*}{ Docentes } & Labor de Enseñanza, Conserjería y Tutoría. \\
\hline & & Labor de Investigación \\
\hline & & Labor de Extensión Universitaria y de Proyección Social. \\
\hline & & $\begin{array}{l}\text { Ambientes y equipamiento para la labor administrativa y } \\
\text { de bienestar. }\end{array}$ \\
\hline & \multirow[t]{2}{*}{$\begin{array}{l}\text { Infraestructura y } \\
\text { Equipamiento. }\end{array}$} & $\begin{array}{l}\text { Ambientes y equipamiento para la enseñanza } \\
\text { aprendizaje e investigación. }\end{array}$ \\
\hline & & $\begin{array}{l}\text { Ambientes y equipamiento para la Extensión Univ. X } \\
\text { Proyección Social. }\end{array}$ \\
\hline & \multirow{2}{*}{$\begin{array}{l}\text { Bienestar } \\
\text { Recursos } \\
\text { Financieros }\end{array}$} & Implementación de programas de Bienestar. \\
\hline & & Financiamiento de la implementación de la Carrera \\
\hline & Grupos de Interés & Vinculación con los grupos de Interés \\
\hline
\end{tabular}

Fuente: CONEAU; 2008, 19

\section{CONCLUSIONES}

1. La universidad peruana, y por ende la administración universitaria, está en un proceso de cambio, en especial, de gran expansión, reflejando los cambios políticos, sociales, culturales y tecnológicos.

2. Para garantizar que la expansión de la oferta universitaria se condiga con las demandas sociales, del mercado y del desarrollo nacional, se han planteado sistemas de gestión de calidad universitaria, a través de los procesos de autoevaluación y acreditación promovidos por CONEAU.

3. Dichos criterios, enfocados como sistemas de gestión de la calidad, incluyen conceptos de planificación estratégica, gestión de la calidad, capital intelectual, formulación de indicadores y son coherentes con el enfoque del Cuadro de Mando Integral.

4. La principal innovación de los Modelos de Calidad de CONEAU es la formulación de indicadores, que permitirán el monitoreo y evaluación acorde con las modernas tendencias administrativas.

5. Es necesario incluir en el proceso una visión prospectiva en el aspecto: científico, industrial, tecnológico, para que la relación Universidad -Ciencia y Cultura este en el centro de la planificación universitaria, como corresponde. 


\section{REFERENCIAS BIBLIOGRÁFICAS}

1.- Ley Universitaria, Ley No 23733, promulgada el 09 de diciembre de 1983, publicada en el Diario Oficial El Peruano el 17 de diciembre de 1983.

2.- CONSEJO DE EVALUACIÓN, ACREDITACIÓN Y CERTIFICACIÓN DE LA CALIDAD DE LA EDUCACIÓN SUPERIOR UNIVERSITARIA. Dirección de Evaluación y Acreditación. Modelo de Calidad para la Acreditación de Carreras Universitarias y Estándares para la Carrera de Educación. Disponible en: www.oei.es/pdfs/modelo_calidad_acreditacion_universitaria.pdf

3.- PERU. Ministerio de Educación. SINEACE Consejo de Evaluación, Acreditación y Certificación de la Calidad de la Educación Superior Universitaria. (CONEAU). Guía para la acreditación de carreras profesionales universitarias del CONEAU El Peruano, miércoles 16 de setiembre de 2009

4.- ASAMBLEA NACIONAL DE RECTORES. COMISIÓN NACIONAL DE RECTORES PARA LA ACREDITACIÓN. DIRECCIÓN GENERAL DE INVESTIGACIÓN Y ACREDITACIÓN UNIVERSITARIA. Modelo De Autoevaluación Con Fines De Mejora De Las Carreras Universitarias. Lima Perú

5.- ASAMBLEA NACIONAL DE RECTORES. Comisión Nacional de Rectores para la Acreditación- Dirección General de Investigación y Calidad Universitaria. GUIA DE AUTOEVALUACION CON FINES DE MEJORA DE LAS CARRERAS UNIVERSITARIAS. Lima, Perú. Octubre 2005

6.- MINEDU. Dirección de Coordinación Universitaria. La Universidad en el Perú. Informe 2006. Lima, 2006. 197 pp. 